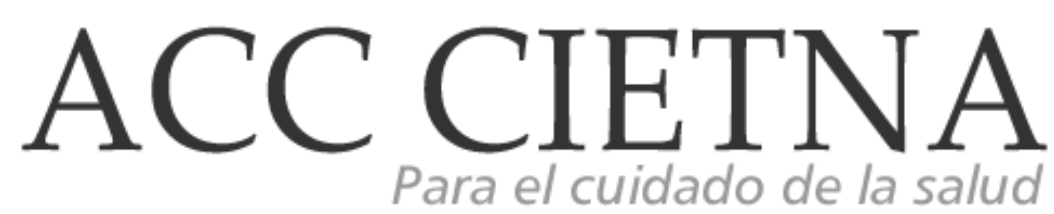

https://doi.org/10.35383/cietna.v4i2.15

ENSAYO

\title{
El diseño curricular por competencias y su impacto en los procesos de enseñanza- aprendizaje de los profesionales de enfermería
}

\author{
Chacón Víquez Luis Diego ${ }^{1}$
}

\begin{tabular}{l} 
INFORMACIÓN DEL ARTÍCULO RESUMEN \\
\hline Historia del artículo:
\end{tabular}

Recibido el 23 de mayo de 2017

Aceptado el 15 de octubre de 2017

\section{Palabras clave:}

Diseño curricular

Competencias

Enseñanza-aprendizaje

Enfermería

Globalización

Educación superior
El presente ensayo corresponde a una revisión bibliográfica profunda de la temática que analizamos. El cambiante y globalizado mundo en el que nos desarrollamos, así como todas las demandas sociales y profesionales a las que nos vemos expuestos hace que las universidades busquen de alguna manera adaptar sus planes de estudio para que sus egresados cuenten con mejores herramientas a la hora de enfrentarse al exigente mercado laboral. La carrera de Enfermería no es la excepción a este caso, por lo que este documento presenta de una manera resumida, lo que implica formar por competencias a los nuevos profesionales de enfermería, cuál es la delimitación conceptual que fundamenta el modelo, cómo incide el modelo, las diferencias del modelo de educación tradicional versus el modelo de educación por competencias, cómo es el diseño curricular por competencias y su impacto en el proceso de enseñanza-aprendizaje de los nuevos profesionales de enfermería, y los cuidados que se debe tener a la hora de implementar el modelo de educación por competencias debido a la polisemia que incide sobre el constructo. Creemos que el modelo de formación por competencias debe implementarse siempre y cuando se realice una revisión profunda y crítica desde una fundamentación teórica y epistemológica por parte de las autoridades, el equipo promotor de la reforma y su cuerpo docente. Es fundamental que el

${ }^{1}$ Docente, Investigador y Consultor en temas educativos y de salud. Licenciado en Enfermería de la Universidad de Costa. Máster en Formación del Profesorado en Docencia e Investigación en la Educación Superior de la Universidad Nacional de Educación a Distancia, España. Candidato a Doctor en Educación, Universidad de Costa Rica, San José, Costa Rica. Email: chaconld@gmail.com 
profesorado de la carrera sea partícipe activo del proceso y conozca de primera mano cómo este cambio incide en su desarrollo profesional.

Competencies based curricular design and its impact on the process of teaching-learning of nursing professionals

\section{ABSTRACT}

\section{Keywords:}

Curricular design

Competencies

Teaching-learning

Nursing

Globalization

Higher education
The present article corresponds to a deep bibliographical revision of the thematic that we analyze. The changing and globalized world in which we develop, as well as all the social and professional demands to which we are exposed, makes the universities somehow seek to adapt their curricula so that their graduates will have better tools when they comes to facing the demanding labor market. The Nursing career is not the exception to this case, so this article presents in a summarized way, what implies to train new nursing professionals by competences, what is the conceptual delimitation that underlies the model, how the model affects, the differences between the traditional education model versus the competency-based education model, how is the curricular design by competencies and its impact on the teaching-learning process of the new nursing professionals, and the care that must be taken at the time of implementing the model of education by competences due to the polysemy that affects the construct. We believe that the competencebased training model should be implemented as long as an in-depth and critical review is carried out from a theoretical and epistemological basis by the authorities and team that promotes the reform. It is essential that faculty members take an active part in the process and know firsthand how this change affects their professional development.

\section{Introducción}

En pleno siglo XXI, la sociedad del conocimiento enfrenta una serie de cambios que se dan a un ritmo acelerado, como nunca sucedió en la historia. Estos cambios se dan desde lo político, lo social, lo científico y lo cultural. Podemos palpar como cambia nuestro contexto con solo analizar el entorno en el que vivimos y darnos cuenta que ya nada es igual a hace 30 o 40 años atrás.

Aún y tomando en cuenta como todo el entorno ha cambiado a un ritmo escalofriante, los modelos educativos aún siguen luchando con la sombra de las formas de enseñar y aprender que se iniciaron con la revolución industrial y que hoy por hoy se repiten en muchos países del mundo.

A partir de buscar nuevas formas de enseñar y aprender, es que desde finales de la década de los años noventa, y producto del cambio en la estructura económica europea, se inició el debate que permitió incursionar en el tema de las competencias en la educación (esto como respuesta a la cada vez mayor utilización del término en el área laboral) situación que facilitó la elaboración de diversos discursos pedagógicos y curriculares en diferentes puntos geográficos del 
planeta, los cuales tuvieron un alcance que llegó hasta la misma reestructuración de los sistemas educativos y la forma en la que se entiende $y$ ejecuta el proceso de enseñanza y aprendizaje, las actividades del profesorado y el papel del estudiantado en su desarrollo académico.

Esta nueva tendencia, centrada en el estudiante ha llevado a los sistemas educativos y a sus docentes a incorporar cambios hacia la consecución de los objetivos de la formación actual de los jóvenes universitarios. Ante esto Bozú \& Cantol plantean que actualmente "el enfoque de la pedagogía centrada en la adquisición y desarrollo de competencias está siendo utilizado en las instituciones de educación superior en el mundo"

A partir del inicio de la era del conocimiento y debido a los requerimientos que se presentan continuamente a nivel académico, social y profesional, la universidad se vuelve fundamental para los procesos de construcción del conocimiento y la generación de las competencias en las diferentes áreas del conocimiento, social y laboral, para que las personas vayan logrando las habilidades necesarias para que su desempeño sea acorde a las demandas del contexto donde se desarrollan.

En este sentido las acciones que se lleven a cabo para propiciar las habilidades en los estudiantes, no pueden desligarse de los procesos que se deben tomar en cuenta para la formación de los docentes que deban poner en práctica todas aquellas acciones que permitan al estudiante construir los conocimientos a partir de la generación paulatina de nuevas competencias para su futuro.

\section{Desarrollo}

\section{La Globalización y su impacto en la educación superior}

Los escenarios económicos, políticos, educativos y sociales internacionales han estado marcados por el embate de la globalización que vino a dictar las pautas del manejo y desempeño de los países. Para entender un poco más el término se define Globalización, según Brunner ${ }^{2}$ como los procesos de movimiento trasnacional de bienes y servicios, además de, personas, inversiones, ideas, valores y tecnologías más allá de las fronteras de los países. Por lo que podemos deducir que la globalización no es una simple reorganización del espacio económico mundial, es un cambio de dimensiones amplias que de una u otra manera obliga a los países a restructurar los mercados laborales y con esto el posible y progresivo debilitamiento de los estados a través de la difusión internacional de los estándares de consumo propios de las sociedades industriales y la aparición de un mercado global.

En una época donde los efectos de la globalización sobre las economías y la educación han influido considerablemente en los cambios socioeconómicos y culturales actuales, los sistemas educativos reconocen como necesarios los cambios que incidan en la educación del Siglo XXI. Para Díaz-Barriga ${ }^{3}$ el efecto de la globalización y sus demandas sobre la educación superior, a través de reformas y cambios en los sistemas educativos centrados en la calidad, la eficiencia y la acreditación se pueden enumerar de la siguiente manera: la política de la educación superior; las diversas estrategias de enseñanza que emanan del desarrollo de la psicología educativa; los planteamientos que surgen de las propuestas curriculares en boga; las concepciones de enseñanza que derivan de la llamada sociedad del conocimiento y de las tecnologías de la información, y las transformaciones en cada disciplina como resultado de un desarrollo permanente de nuevos espacios de conocimiento.

Por otra parte, la globalización ha traído consigo procesos de interconexión entre personas que no se dieron anteriormente en la historia. Hoy en día el número de usuarios que tienen acceso a las Tecnologías de la Información y la Comunicación (TIC) mediadas por el internet es amplio, generando con esto propensión a contar con 
mayores flujos de información y de herramientas para la generación de nuevo conocimiento.

Este poder denominado como conocimiento, es poco equitativo e inclusivo en el caso particular de América Latina (segunda región más desigual del planeta con un 52,9 de coeficiente de Gini y con un nivel de pobreza que afectó al $23.3 \%$ de la población), debido a que es un derecho para unos pocos, si hacemos la relación con el alto porcentaje de personas de la región vive con menos de un dólar diario.

Por lo tanto, la red de interconexión está lejos de ser un espacio de encuentro para todas las personas, ya que cada país vive su realidad de diferente manera enfrascado en su deficiencia tecnológica, de conocimiento y por qué no decirle de educación adaptada a los cambios presentes. Ante estas afirmaciones y relacionados con lo que Kim Anh \& Marginson ${ }^{4}$ nos plantean que panorama en el que:

Much contemporary 'globalization' discourse is ideological, designed to render 'natural' and 'inevitable' the hegemony of Anglo-American systems and methods in government, the economy and universities (...) Education is thoroughly implicated in these contrary potentials. It is impacted by economic globalization via markets in student places, trade in educational goods and foreign for-profit providers that operate inside national systems with the sanction of the World Trade Organization's General Agreement on Trade in Services"

Es la emergente sociedad de la información, con sus contradicciones y brechas a nivel global -y no la globalización como proceso histórico más general ni las nuevas tecnologías consideradas al margen de la sociedad- la que proporciona el entorno más inmediato donde se desarrollarán los nuevos escenarios educacionales. ${ }^{2}$

Debido a la globalización y a los aspectos socioculturales a los que estamos expuestos constantemente, los objetivos esenciales en los que se basa el aprendizaje se ven modificados, aunque no a la velocidad que los avances en el conocimiento se van dando, en gran parte, debido a los cambios drásticos que se viven en ámbitos relacionados con la investigación, generación de nuevos conocimientos y el respectivo acercamiento de otras personas a conocimientos diversos que terminan por influir en el perfil de egreso de los nuevos profesionales.

\section{Delimitación conceptual de las competencias}

Es importante mencionar que las competencias como tales, no nacen de una necesidad de las instituciones educativas, sino más bien, aparecen relacionadas con los procesos productivos de las empresas, especialmente en los campos ligados al desarrollo tecnológico, donde es conocido su explosivo crecimiento hasta el día de hoy.

Pero no es sino hasta hace pocas décadas que la discusión de lo que hoy llamamos competencias se realizó desde un punto de vista de la lingüística con el fin de explicar el carácter creativo o generativo del lenguaje del ser humano, para poco a poco pasar a ser reconocido como las características del desempeño de una persona a la hora de resolver determinado problema. Aunque el concepto competencia aparece en la década de los setentas y tiene como referente las investigaciones de McClellan; por otro lado Chomsky, buscaba explicar el carácter creativo o generativo del lenguaje humano, mediante la apropiación del sistema lingüístico de los niños y niñas.

Para McClellan, según Campos y González 5 los test de inteligencia, de conocimiento y de aptitud así como las pruebas tradicionalmente utilizadas no se correlacionaban con el rendimiento en un puesto de trabajo o en una determinada situación. Así mismo, McClelland delimitaba las competencias como características subyacentes en una persona y que se correlaciona con la mayor eficiencia y rendimiento en el desempeño de un puesto de trabajo, una función laboral o una situación.

Tobón 6 , haciendo referencia a Chomsky, utiliza el concepto de competencia lingüística como una 
estructura mental implícita y genéticamente determinada que se pone en acción mediante el desempeño comunicativo (uso efectivo de la capacidad lingüística en situaciones específicas), por lo que siempre opone el marco de su gramática generativa transformacional competenciasdesempeño. "A partir de esto, el concepto de competencias comenzó a tener múltiples desarrollos, críticas y reelaboraciones, tanto en lingüística como en la psicología (conductual y cognitiva) y en la educación"

A raíz de los trabajos realizados por Chomsky y McClellan surgió, en la década de los setentas, lo que Cejas $^{7}$ menciona como el movimiento "Enseñanza basada en competencias", que se fundamentó en cinco principios:

- Todo aprendizaje es individual.

- El individuo, al igual que cualquier sistema, se orienta por las metas a lograr.

- El proceso de aprendizaje es más fácil cuando el individuo sabe qué es exactamente lo que se espera de él.

- El conocimiento preciso de los resultados también facilita el aprendizaje.

- Es más probable que un alumno haga lo que se espera de él y lo que él mismo desea, si tiene la responsabilidad de las tareas de aprendizaje.

Pero cabe mencionar que conforme han pasado los años, muchas de las características que han traído consigo el concepto de las competencias se han ido incorporando a las instituciones de Educación Superior desde una perspectiva de corte más holista que facilite la incorporación de los egresados y egresadas al ámbito laboral.

Partir de una formación profesional que además de promover el desarrollo de ciertos atributos (habilidades, conocimientos, actitudes, aptitudes y valores), considere la ocurrencia de varias tareas (acciones intencionales) que suceden simultáneamente dentro del contexto (y la cultura del lugar de trabajo) en el cual tiene lugar la acción; y a la vez permita que algunos de estos actos intencionales sean generalizables" 8
Ya para finales de la década de los noventa y hasta la fecha, en el campo de la educación se han venido tocando temas relacionados con las competencias dentro de las que destacan la formación por competencias, planes de estudio basados en el enfoque por competencias, propuestas educativas por competencias, evaluación de las competencias docentes y competencias docentes. Por lo que el tema de centralización de dicha temática se da como una alternativa para las instituciones educativas, sus docentes y sus estudiantes.

Ello ha llevado a que la literatura sobre este tema se haya incrementado recientemente y en la cual se presentan diversas interpretaciones relacionadas con esta noción, se establecen algunas alternativas para poderla emplear en diversos ámbitos de la formación escolar, tales como la educación básica, la formación del técnico medio y la formación de profesionales con estudios de educación superior.

\section{El contexto de las competencias en educación}

Desde la perspectiva de Mas9 ${ }^{9}$, el desarrollo no ha sido el mismo en los diferentes escenarios donde se ha buscado implementar la reforma educativa que orienta el sistema educativo hacia las competencias. Se encuentran algunos casos donde el nivel de avance ha sido mayor, como lo fue la reforma que sufrió el Espacio Europeo de Educación Superior hacia la formación de profesionales con competencias definidas, esto a raíz de la Declaración de la Sorbona, que generó posteriormente la Declaración de Bolonia.

La Declaración de Bolonia propuso "armonizar los distintos sistemas educativos y proporcionar una forma eficaz de intercambio

de estudiantes, docentes y diplomados; así como dotar de una dimensión y de una agilidad sin precedentes al proceso de cambio emprendido por las universidades europeas". 10 Se establecieron unos objetivos que buscan de alguna manera delimitar los escenarios educativos, a través de la homologación y convergencia europea que conlleva la creación de un sistema que busca tener 
una mayor transparencia y transferibilidad de sus procesos en un nuevo diseño de titulaciones definidas en términos de competencias $y$ habilidades, una organización y gestión curricular orientada al aprendizaje activo del estudiante y una dimensión internacional de los estudios basada en la flexibilidad curricular, todo a través del programa denominado Tuning en Europa.

El Espacio Europeo de Educación buscó armonizar los diferentes sistemas universitarios para que los estudios tengan un sistema estructural igual entre los países, para contribuir con ello a promover lo que denominan la "economía del conocimiento". 10 Por otra parte, tal y como lo menciona el Parlamento Europeo (2006), se pretendía aprovechar la diversidad en las competencias individuales, por lo que "se debería responder a las diferentes necesidades de los alumnos garantizando la igualdad de acceso para aquellos grupos que, como consecuencia de desventajas educativas causadas por circunstancias personales, sociales, culturales o económicas, necesiten un apoyo especial para desarrollar su potencial educativo".

Para el caso de América Latina los esfuerzos por llevar a cabo la implementación del Tuning se iniciaron en Buenos Aires Argentina en el 2004, como respuesta a las demandas de los empleadores que pedían un recurso humano con una formación que diera respuesta a las necesidades del entorno, de la empresa y de la producción de los países. "El Proyecto Tuning, nacido en el espacio de la Educación Superior por el trabajo conjunto de más de 176 universidades europeas, en un intento de buscar metas comunes que den continuidad a la Declaración de Bolonia". 11 Como parte del trabajo producto del Tuning, se elaboró la lista de competencias genéricas que se consultarían a académicos, estudiantes, graduados y empleadores. De esta forma, el punto de partida del proyecto estaría en la búsqueda de puntos de referencia comunes, centrándose en las competencias y en las destrezas (basadas siempre en el conocimiento).

\section{La formación de profesionales en enfermería}

Al realizar una revisión bibliográfica, encontramos que es poca la información relacionada con programas de formación profesionales de enfermería desde la metodología por competencias.

Por lo tanto, creemos necesario que los programas de formación de profesionales en enfermería, al igual que otras áreas del conocimiento, requieren de una revisión constante, sistemática, y acorde a las necesidades que los diferentes empleadores, fundamentados en estudios de tipo demográficos, epidemiológicos y sociales.

A fin de satisfacer estas necesidades se debe desarrollar en el estudiante el empoderamiento como una forma visible de identidad profesional, para que se facilite la adquisición de poder, autonomía y responsabilidad y se cumpla con las demandas de la sociedad y con la prestación de los servicios.

Para Jiménez ${ }^{12}$, es conveniente señalar que la profesión de enfermería, a diferencia de otras disciplinas, se forma básicamente en tres niveles académicos: medio superior, licenciatura y posgrado.

Acorde con Villar, et al13, la formación en Enfermería ha dependido, en gran medida, de la época y contexto en que se encuentre, de la situación que vive la sociedad; de las necesidades de contratación, la perspectiva teórica con relación a la Enfermería, las tendencias en la atención a la salud y en la educación, entre otras cosas, con el fin de formar un profesional con mayores y más amplios conocimientos en diversas áreas.

Desde la óptica de Plaza \& Soriano ${ }^{14}$, la formación en Competencia Cultural forma parte de los estudios de grado y postgrado de Enfermería en países como Estado Unidos, Canadá, Reino Unido o Australia, entre otros. En estos países existen algunos hospitales en los que incluso se llegan a desarrollar entrenamientos específicos en 
competencia intercultural para profesionales $y$ sólo se seleccionan aquellos que poseen formación específica en este campo, como es el caso de la ciudad de Nueva York.

Según González, et al15 "Las instituciones educativas, tanto universitarias como de formación de profesionales generales y técnicas en España y México, han promovido el uso del Proceso Enfermero como método de trabajo. Hoy en día, la mayoría de las enfermeras técnicas y las licenciadas en enfermería conocen parcial o totalmente este método".

Achury ${ }^{16}$ plantea que la educación en enfermería debe ser asumida estimando un trabajo en equipo institucional, que permita utilizar y desarrollar recursos de aprendizaje semejantes a los requerimientos actuales de la sociedad, aprovechando la actividad científico-tecnológica y empleando todo el potencial de los medios existentes. Esto involucra la creación de ambientes propicios para el crecimiento individual de los estudiantes y el desenvolvimiento académico, para que favorezcan una formación profesional activa, creativa y critica.

\section{El modelo de educación tradicional versus el modelo de educación por competencias}

Cuando tratamos de comparar el modelo de educación tradicional contra el modelo de educación por competencias, encontraos diferencias significativas y que permiten de alguna manera que los estudiantes se logren adaptar más al contexto laboral y social actual.

Desde el modelo de enseñanza tradicional el aprendizaje se produce a partir del contenido de las disciplinas de una manera abstracta, no siempre contextualizada, mientras que en el modelo de educación por competencias el aprendizaje se da cuando los procesos cognitivos $y$ afectivos desencadenan los procesos metacognitivos que llevan a la resolución de una tarea específica, a través del aprendizaje situado en los diferentes contextos donde se encuentre la persona.
El modelo de enseñanza tradicional promueve el aprendizaje memorístico e individual, aquel que es muy común ver aún hoy en día en nuestras escuelas, colegios y universidades y que permite a los estudiantes aprobar sus exámenes, sin que esto impacte directamente sobre la metacognición. El modelo de educación por competencias busca propiciar aprendizaje de tipo significativo y funcional desde un punto de vista colaborativo, donde se da una sinergia entre el docente y los estudiantes.

Por otra parte, en el modelo tradicional el profesorado juega un papel de transmisor de conocimientos, muy común aun en nuestros días donde el aprendizaje se hace centrado en el docente. En el modelo de educación por competencias el docente pasa a ser un guía, un facilitador de los conocimientos que apoya al estudiante a la hora de construir los suyos. Además este modelo se centra más en el estudiante y el docente pasa a tener un papel de apoyo.

El modelo imperante hoy en día, el tradicional que viene desde la misma revolución industrial y que tuvo como fundamento formar a las personas para que fueran operarios de las grandes compañías, sigue preparando a los estudiantes para la vida académica, para que se encuentre preparado para el siguiente grado escolar, más sin embargo, se hace de una manera descontextualizada con la realidad en la que viven las personas. En el modelo de educación por competencias, se busca formar desde una perspectiva holísticas a personas para que puedan enfrentar y encajar en los contextos donde se desarrollen, desde una visión de preparación para la vida.

Ante lo que hemos analizado, el modelo de educación por competencias viene a ser una alternativa ante un modelo tradicional imperante en muchos centros de educación superior de América Latina, y que si bien es cierto encuentra muchos detractores, aconsejamos que el modelo sea analizado, socializado, investigado y llegue a ser comprendido por todos los participantes 
(Rectoría, Direcciones Académicas, Jefaturas, Docentes, Estudiantes, Administración) para que a la hora de la ejecución a través del diseño curricular por competencias se puedan identificar las particulares del contexto y sea más fácil llevar a la implementación, esto sin olvidar, que es necesaria la fundamentación epistemológica $y$ teórica, un adecuado proceso de socialización y de formación de las partes involucradas, pues de no hacerse de esta manera se corre el riesgo de tener un diseño curricular por competencias en el papel, pero un modelo tradicional con tintes conductistas en la ejecución, pues si algo nos queda claro es que la planificación y evaluación de los aprendizajes no puede ser igual al modelo tradicional.

A manera de resumen presentamos la figura I, expuesta por Falcó. ${ }^{17}$

\section{El diseño curricular por competencias y su impacto en el proceso de enseñanza-aprendizaje}

Guzmán, González y Marín ${ }^{18}$, plantean que los docentes universitarios requieren contar con al menos tres competencias (interacción pedagógica, comunicación educativa y evaluación de los aprendizajes) que corresponden a la función y práctica del docente dentro del aula, situación que se vuelve compleja con el panorama de falta de capacitación al que se refieren Beneitone, et al.19

Desde la óptica de Guzmán 20 el diseño curricular cualquier carrera, refleja esta forma particular de pensar la profesión, comprender la realidad, de mirar y tratar de comprender a los otros, de relacionarse con la sociedad, de entender la educación de personas y el mejor modo de aprender la disciplina, todo esto mediado por el o la docente. Nos queda claro que podemos afrontar cualquier forma de pensamiento.

Para Pantic' \& Wubbels 21 el panorama de las competencias en educación ha encontrado diversos detractores ya que un buen docente no puede ser descrito en términos de habilidades aisladas, ya que dicha fragmentación no tiene en cuenta los aspectos de la personalidad de los profesores que desempeñan un papel crucial en la enseñanza eficaz. Ante esto es que se hace esencial conocer cómo percibe y construye el profesorado las competencias docentes, más allá de un marco rígido y limitado.

Across the world, community expectations for teacher quality appear to be rising at the same time as the status of teachers is falling (...) Teachers need to possess a body of knowledge and be able to apply that knowledge to a variety of situations within their professional setting.

Desde el punto de vista de Corcino 22 es observable con frecuencia que el profesor se limita a emplear un número reducido de estas representaciones; relacionadas con las múltiples definiciones del concepto de función que conoce y manipula el profesor, en qué orden las enseña y como relaciona unas con otras; privando al estudiante de la oportunidad de profundizar sus estructuras conceptuales. "El objetivo principal de todo docente debe ser proveer al estudiante de un entorno pedagógico adecuado que estimule el desarrollo y la construcción paulatina del conocimiento."

Este panorama, donde cada vez son más necesarias las competencias docentes adaptadas a las demandas del medio, según Serdenciuc ${ }^{23}$, resulta en un replanteamiento de la formación del profesorado ya que "Dealing with a new perspective of knowledge management in a global economy, education systems must evolve focusing on rethinking the teaching-learning process in order to prepare better individuals for meeting the changing social and economic demands" Ante esto, la universidad como tal junto con el docente universitario, les corresponde proveer al estudiante espacios con la complejidad de las situaciones del mundo real, lo que requiere una consolidación de ciertas competencias por parte del profesorado que conlleven un desempeño eficiente y eficaz a través de la construcción, más centrada en el estudiante, de competencias básicas y competencias específicas. 
In higher education, approaches to teaching are becoming more student-centered, which demands different teaching competencies. Therefore, it is necessary to have an adequate framework of teaching competencies that can be used for evaluation purposes. The weaknesses of the existing frameworks are that they do not pay attention to the person as teacher, they are too narrowly defined, they are not validated and they are not adjusted to modem approaches to teaching. ${ }^{24}$

El panorama cambiante de la globalización y el efecto directo e indirecto que tiene sobre cómo y qué se debe enseñar sigue cambiando día con día ya que para Serdenciuc ${ }^{23}$ "The expanded access to learning opportunities, for the potential beneficiaries, beyond the traditional way of delivered school instruction, sustains an educational approach focused on competencies, as a result of the integrative learning experiences, bounding skills, abilities and knowledge in combinations used for efficient task performing", lo que sin duda alguna tiene efectos significativos sobre la labor docente y la manera en la que construye el conocimiento junto con sus estudiantes.

Al mismo tiempo, la presión social y profesional en los sistemas educativos se refleja considerablemente en el uso indiscutible de las TIC y laboratorios virtuales como herramientas de aprendizaje, algo que no se visualizaba completamente a inicios de este siglo, tal y como lo menciona Lucas 25 pues para la autora "Educators and administrators are beginning to realize that technology should not be used as a tool for efficiency, but as a tool for learning. Additionally, the ability and potential that technology has to address issues of educational inequity in terms of among other things, learning styles, are becoming more and more apparent.

\section{Conclusiones}

Los contextos sociales, culturales y laborales están cambiando a un ritmo vertiginoso. La universidad, como ente de formación de nuevos profesionales no puede cerrar sus puertas ante las nuevas tendencias curriculares existentes, por lo que debe analizar, con una mente crítica, las implicaciones y razones que le llevan a buscar cambios significativos en los currículos de las carreras que imparte y que sin duda alguna implique el desarrollo integral y holístico de sus estudiantes. El modelo de educación por competencias, al tener el gran problema de la polisemia, debe ser analizado, comprendido y llevado a la reflexión por todas las instancias de la universidad, desde la rectoría, hasta los estudiantes y empleadores, pasando sin lugar a dudas por su cuerpo docente, que al final de cuentas es que ejecuta los procesos de enseñanza y aprendizaje. Se debe estar consciente de la fundamentación epistemológica y teórica del modelo para que así exista la claridad necesaria de cómo se van a desarrollar las competencias plasmadas en los planes de estudio y de qué manera serán construidas por el estudiantado de la mano de cada uno de los docentes.

La sociedad actual demanda de profesionales mejor capacitados, con más habilidades y competencias que anteriormente no se pedían o no se identificaban de manera individual. Los programas de estudio de profesionales en enfermería deben de buscar afianzar aquellos modelos curriculares que permitan al estudiantado conformar las habilidades requeridas para el desarrollo de los principios fundamentales del cuidado y la teoría de enfermería, que son los que dan el fundamento a la profesionalización de la enfermería. Creemos que un modelo de educación por competencias debe, sin lugar a dudas, mantener esos saberes fundamentales de la enfermería, pero vistos desde una visión global, centrada en la atención de las personas y en las múltiples situaciones que surgen cuando de la salud de las personas se trata, pero sin dejar por fuera el saber, saber hacer, saber ser y saber convivir.

Al igual que en otras profesiones, la implementación del modelo de formación por 
competencias en la enfermería, es una opción que busca preparar y adaptar a sus egresados ante las demandas actuales del entorno, pero sin perder de vista los requerimientos y necesidades sociales, laborales, del cuidado y profesionales. Si una escuela o facultad de Enfermería busca desarrollar este modelo curricular, esto implica que se debe redefinir el proyecto educativo, además de socializar, formar y promover acciones pedagógicas y didácticas entre su profesorado que se traduzcan en resultados palpables en sus egresados.

Es imperante que para que un modelo de educación por competencias se logre implementar, se deben desarrollar acercamientos y alianzas con las organizaciones civiles y laborales que permitan tener una visión más global y contextualizada de lo que debe ser un profesional en enfermería en el siglo XXI. Esto facilitará mucho la ejecución del proceso de enseñanza y aprendizaje que el profesorado pueda llegar a ejecutar, por lo que sin duda alguna, los docentes no pueden quedar, bajo ninguna circunstancia, fuera del proceso de diseño e implementación curricular.

\section{Referencias bibliográficas}

1. Bozú Z, Canto, P. El profesorado universitario en la sociedad del conocimiento: competencias profesionales docentes. Revista de Formación e Innovación Educativa Universitaria. 2009. Vol. 2, $\mathrm{N}^{\circ}$ 2, 87-97. España. Disponible en:http://tecnologiaedu.us.es/mec2011/h $\mathrm{tm} / \mathrm{mas} / 3 / 31 / 26 . p d f$

2. Brunner J. Globalización y el futuro de la educación: Tendencias, desafíos, estrategias. Seminario sobre Prospectiva de la Educación en la Región de América Latina y el Caribe. 2000.

3. Díaz-Barriga A. El profesor de educación superior frente a las demandas de los nuevos debates educativos. Perfiles educativos, 2005; 27(108), 9-30. Recuperado http://www.scielo.org.mx/scielo.php?scri $\mathrm{pt}=$ sci_arttext $\&$ pid $=$ S018526982005000100002\&lng=es\&tlng=es

4. Kim Anh D, Marginson S. Global learning through the lens of Vygotskian sociocultural theory. Critical Studies in Education, 2013; 54:2, 143-159. Disponible en: http://dx.doi.org/10.1080/17508487.20 $\underline{12.722557}$

5. Campos A, González D. Elaboración de un instrumento docente para la evaluación de las competencias en las asignaturas de dirección y organización en el grado de ciencias del deporte. Univest 09. 2009. Disponible en: http://dugidoc.udg.edu:8080/bitstream/h andle $/ 10256 / 1942 / 205 . p d f$ sequence $=1$

6. Tobón S. Aspectos básicos de la formación basada en competencias. Talca: Proyecto Mesesup. 2006. Disponible en:

http://maristas.org.mx/gestion/web/doct os/aspectos_basicos_formacion_competen cias.pdf

7. Cejas E. La formación por competencias laborales: proyecto de diseño curricular para el técnico medio en farmacia Industrial. [Tesis Doctoral]. ISPEJV, Ciudad de La Habana, Septiembre, 2005, Disponible en: www.monografias.com/trabajos $40 /$ formac ion-por-competencias/formacion-porcompetencias.shtml

8. Huerta J, et al. Desarrollo curricular por competencias profesionales integrales. Sin fecha. Disponible en: http://educacion.jalisco.gob.mx/consulta Leducar/13/13Huerta.html

9. Mas O. El profesor universitario: sus competencias y formación. Profesorado. Revista de curriculum y formación del profesorado. 2011. Disponible en: http://www.ugr.es/local/recfpro/rev153C OL1.pdf 
10. Porta E. Tratado de Bolonia Convergencia de los sistemas de enseñanza superior europea. edUTecNe -Editorial de la Universidad Tecnológica Nacional. 2009. Disponible

en: http://www.edutecne.utn.edu.ar

11. Flores F. Las competencias que los profesores de educación básica movilizan en su desempeño profesional docente. [Tesis Doctoral]. Facultad De Educación. Departamento de Psicología Evolutiva y de la Educación. Universidad Complutense de Madrid, España. 2008.

Disponible

en: http://eprints.ucm.es/8171/1/T30412.pd f

12. Jiménez J. Formación de recursos humanos en enfermería. La calidad de la atención a la salud en México. Sin fecha. Disponible en:

http://www.calidad.salud.gob.mx/site/edi torial/docs/dgr-editorial_01M.pdf

13. Villar A, et al. Tendencias en la formación y desarrollo de los profesionales de la Enfermería en la ENEO. Revista Enfermería Universitaria ENEO-UNAM. 2007. Vol 4. No.

2 Año 4 Mayo- Agosto. Disponible en: www.revistas.unam.mx/index.php/reu/art icle/download/30294/28140

14. Plaza F, Soriano E. Formación de los profesionales de enfermería: Cuidar en la sociedad multicultural del siglo XXI. Revista Scielo. 2009. Disponible en: http://scielo.isciii.es/scielo.php?script=sci _arttext\&pid $=$ S1132_ 12962009000300011

15. González E, et al. La profesionalización en la enfermería: Un proceso de cambio. Tecnociencia, Chihuahua. Vol VI, No 1. Enero-abril, 2012. Disponible en: http://tecnociencia.uach.mx/numeros/v6 n1/data/La_profesionalizacion_en_enferm eria_hacia_una_estrategia_de_cambio.pdf

16. Achury D. Estrategias pedagógicas en la formación de profesionales de enfermería.
Investigación en Enfermería: Imagen y Desarrollo - Vol. $10 \mathrm{~N}^{\circ}$ 2. 2008. Disponible en:

https://www.google.com.mx/url?sa=t\&rct $=\mathrm{j} \& \mathrm{q}=$ \&esrc $=\mathrm{s} \&$ source $=$ web\&cd $=18 \& \mathrm{cad}$ $=$ rja\&uact $=8 \&$ ved $=0$ ahUKEwim $3 v 3 Q 543 T$ AhULh1QKHY19D844ChAWCEYwBw\&url=h ttp\%3A\%2F\%2Frevistas.javeriana.edu.co\%2 Findex.php\%2Fimagenydesarrollo\%2Farticl e\%2Fdownload\%2F1604\%2F1027\&usg=AF QjCNFeft3B8yDYSuWVkt|cXJ7LTdYTsg\&sig $2=$ =PdAuu20aMTXzS_Cyui57UA\&bvm=bv. 1 51426398,d.eWE

17. Falcó A. La nueva formación de profesionales: sobre la competencia profesional y la competencia del estudiante de enfermería. Educación Médica 2004; 7(1): 42-45. Disponible en: http://scielo.isciii.es/pdf/edu/v7n1/origin al4.pdf

18. Guzmán I, González A, Marín R. Evaluación de competencias docentes en posgrados de instituciones formadoras de docentes. Revista Iberoamericana de Evaluación Educativa 2010 - Volumen 3, Número 1e. Disponible en:

http://rinace.net/riee/numeros/vol3num1_e/art20.pdf

19. Beneitone P, et al. Reflexiones $y$ perspectivas de la Educación Superior en América Latina. Informe final -Proyecto Tuning- América Latina 2004-2007. Universidad de Deusto, Universidad de Gronigen. 2007.

20. Guzmán A. Gestión curricular: la experiencia de la Licenciatura en Enfermería de la Universidad de Costa Rica. Revista Enfermería actual en Costa Rica. 2013. Vol. 24.

21.Pantic' N, Wubbels T. Teacher competencies as a basis for teacher education - Views of Serbian teachers and teacher educators. Elsevier Ltd. Department of Pedagogical and Educational Sciences, Utrecht University. 
The Netherlands. 2009. Disponible en: http://dspace.library.uu.nl/handle/1874/ 252985

22. Corcino L. Concepciones que orientan la enseñanza-aprendizaje del concepto de función de profesores universitarios. [Tesis doctoral]. Facultad de Educación Universidad de Puerto Rico, Recinto de Rio Piedras. UMI Microform 3314515 by ProQuest. 2008. Disponible en: http://search.proquest.com.ezproxy.sibdi. ucr.ac.cr:2048/pqdtft/docview/30442718 6/fulltextPDF/14261D56173190065B4/4? accountid $=28692$

23. Serdenciuc N. Competency-Based Education - Implications on Teachers' Training. Elsevier Ltd. Teacher Training Department, Stefan cel Mare University, Romania. 2013. Disponible en: http://www.sciencedirect.com/science/art icle/pii/S1 $87704281300743 \mathrm{X}$

24. Tigelaar D, et al. The Development and Validation of a Framework for Teaching Competencies in Higher Education. Kluwer Academic Publishers. Department of Educational Development and Research, University of Maastricht. The Netherlands. 2004. Disponible en: http://link.springer.com/article/10.1023/ B:HIGH.0000034318.74275.e4

25. Lucas S. Who am I? The influence of teacher beliefs on the incorporation of instructional technology by higher education faculty. [Tesis doctoral]. Department of Educational Leadership, Policy and Technology Studies in the Graduate School of The University of
Alabama. UMI Microform 3193807 by ProQuest. 2005. Disponible en: http://search.proquest.com.ezproxy.sibdi. ucr.ac.cr:2048/pqdtft/docview/30502242 1/fulltextPDF/14262104AC461E422F/1?a ccountid $=28692$ 